\title{
Phase Characterization of Cucumber Growth: A Chemical Gel Model
}

\author{
Bo Li, ${ }^{1,2}$ Xuejing Liu, ${ }^{1,2}$ and Junshi Zhang ${ }^{2,3}$ \\ ${ }^{1}$ School of Mechanical Engineering, Xian Jiaotong University, 28 Xianning West Road, Xian 710049, China \\ ${ }^{2}$ State Key Laboratory for Strength and Vibration of Mechanical Structures, Xian Jiaotong University, 28 Xianning West Road, \\ Xian 710049, China \\ ${ }^{3}$ School of Aerospace, Xian Jiaotong University, 28 Xianning West Road, Xian 710049, China \\ Correspondence should be addressed to Bo Li; blancolee@gmail.com
}

Received 15 December 2015; Accepted 22 February 2016

Academic Editor: Kai Chen

Copyright (C 2016 Bo Li et al. This is an open access article distributed under the Creative Commons Attribution License, which permits unrestricted use, distribution, and reproduction in any medium, provided the original work is properly cited.

Cucumber grows with complex phenomena by changing its volume and shape, which is not fully investigated and challenges agriculture and food safety industry. In order to understand the mechanism and to characterize the growth process, the cucumber is modeled as a hydrogel in swelling and its development is studied in both preharvest and postharvest stages. Based on thermodynamics, constitutive equations, incorporating biological quantities, are established. The growth behavior of cucumber follows the classic theory of continuous or discontinuous phase transition. The mechanism of bulged tail in cucumber is interpreted by phase coexistence and characterized by critical conditions. Conclusions are given for advances in food engineering and novel fabrication techniques in mechanical biology.

\section{Introduction}

Development of fruit growth can be identified as a series of successive stages. Before harvest, it develops nonlinearly, then enlarges slowly at initial period, and then grows rapidly as it approaches its maturation. At the moment of harvesting, the fruit is not fully ripened but leaves space for growth that may continue during agricultural storage and transportation for sale. This explains why bananas are almost green and stiff on the shelves of a supermarket and then turn yellow and soft gradually in your kitchen. The growth of fruit after harvest is denoted as postharvest behavior. Ethylene is frequently used to accelerate the process of ripening [1].

According to recent TV news, some cucumbers in the kitchen displayed inhomogeneous deformation in body and their tails bulged locally, as seen in Figure 1. Concerns for the food safety were raised about this unusual phenomenon, since the underlying mechanism, relating multifields knowledge in chemistry, biology, and mechanics, was elusive. In cross-disciplines of plant study, enormous efforts have been devoted to the research of plant growth, including experts from botany to mathematics $[2,3]$. In solid mechanics, the establishment of the nonlinear gel theory offers a new insight to the study of biomechanics. Using the gel theory, the quantities in physics, chemistry, and biology are coupled [4]. Since both gel and biological tissues are soft, flexible, and stretchable, the expansion of biological organs under the regulation of hormones is analogous to the swelling of gel. Large deformation and mass transportation of gel are capable of characterizing the biological system, that is, the morphology of esophagi in growth [5], pattern formation of pumpkin shell [6], wrinkle profile of a finger immersed in water [7], dehydrated green pea in a dry environment [8], and so forth. Through these researches, the capability of gel theory has been reproduced and verified by modeling in biology. In the current presentation, we also adopt the gel theory to analyze the growth process in a specific fruit category: the cucumber. The phenomenon in growth resembles the phase transition in classic thermodynamic systems, while the biochemistry is involved.

\section{A Chemomechanical Model}

Figure 2 sketches an illustration of a thermodynamic system for gel. We first model the growth of a cucumber before 


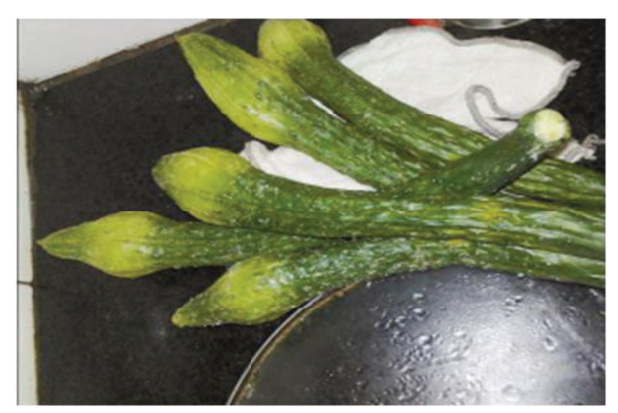

FIgURE 1: Growth of cucumber is complex. After harvest, the cucumbers are immature and ripen gradually during storage. This phenomenon is defined as postharvest ripening. During postharvest, as a result of biological nature, some cucumber species select to ripen part of the whole body, with limited nutrition. Therefore, they show a local inhomogeneous growth at the tail of a cucumber body. Report by Phoenix TV: http://v.ifeng.com/news/society/ 201205/05a4eed1-dc0b-4936-b3be-fb70681691f9.shtml.

harvest as a process of swelling in a cylinder gel. The dry polymer network imbibes solvent molecules (individual volume of $v$ ) and enlarges itself from volume $\pi R^{2} \times L$ at initial state to volume $\pi r^{2} \times l$ at harvested state. In fruit development, the hormones stimulate the expansion of cells, tissues, and organs, which is similar to the swelling mechanism of gel under its chemical potential $\mu$.

$W\left(\lambda_{1}, \lambda_{2}, \lambda_{3}, \mu\right)$, the free energy per unit initial volume of the gel, is the combination of $W_{s}$, the strain energy in polymer network due to stretching, and $W_{m}$, the chemical energy due to mixing network with solvent $[4,9]$. Consider

$$
W\left(\lambda_{1}, \lambda_{2}, \lambda_{3}, \mu\right)=W_{s}+W_{m} .
$$

Here, $\lambda_{i}, i=1,2,3$ are the principle stretches to be determined by coordinates. The free energy expression is then specified by Gent model [10] and Flory-Rehner function [11] as

$$
\begin{aligned}
W_{s}= & -\frac{N K T}{2} J_{m} \log \left(1-\frac{\lambda_{1}^{2}+\lambda_{2}^{2}+\lambda_{3}^{2}-3}{J_{m}}\right) \\
& +\frac{N K T}{2}\left(\frac{J^{2}-1}{2}-\log J\right)^{4}, \\
W_{m}= & -\frac{K T}{v}\left[(J-1) \log \frac{J}{J-1}+\frac{\chi}{J}\right]-\frac{\mu}{v}(J-1),
\end{aligned}
$$

where $N$ is the number of crosslinks in unit initial volume, $K$ is the Boltzmann constant, $T$ is the temperature, $J_{m}$ is the maximum extension limit, $\chi$ is a dimensionless constant, $C$ is the concentration of solvent molecule in initial volume, and $J=1+\nu C=\lambda_{1} \lambda_{2} \lambda_{3}$.

According to the thermodynamics, we define the nominal stresses as $s_{1}=\partial W\left(\lambda_{1}, \lambda_{2}, \lambda_{3}, \mu\right) / \partial \lambda_{1}$, $s_{2}=\partial W\left(\lambda_{1}, \lambda_{2}, \lambda_{3}, \mu\right) / \partial \lambda_{2}$, and $s_{3}=\partial W\left(\lambda_{1}, \lambda_{2}, \lambda_{3}, \mu\right) / \partial \lambda_{3}$. Then we have

$$
\begin{aligned}
& \frac{s_{1}}{K T / v}=N v\left[\frac{\lambda_{1}}{1-\left(\lambda_{1}^{2}+\lambda_{2}^{2}+\lambda_{3}^{2}-3\right) / J_{m}}\right. \\
& +\frac{2}{\lambda_{1}}\left(\left(\lambda_{1} \lambda_{2} \lambda_{3}\right)^{2}-1\right) \\
& \left.\cdot\left(\frac{\left(\lambda_{1} \lambda_{2} \lambda_{3}\right)^{2}-1}{2}-\log \lambda_{1} \lambda_{2} \lambda_{3}\right)^{3}\right]+\frac{1}{\lambda_{1}}[1 \\
& +\lambda_{1} \lambda_{2} \lambda_{3} \log \left(1-\frac{1}{\lambda_{1} \lambda_{2} \lambda_{3}}\right)+\frac{\chi}{\lambda_{1} \lambda_{2} \lambda_{3}}-\frac{\mu}{K T} \\
& \left.\cdot \lambda_{1} \lambda_{2} \lambda_{3}\right], \\
& \frac{s_{2}}{K T / v}=N v\left[\frac{\lambda_{2}}{1-\left(\lambda_{1}^{2}+\lambda_{2}^{2}+\lambda_{3}^{2}-3\right) / J_{m}}\right. \\
& +\frac{2}{\lambda_{2}}\left(\left(\lambda_{1} \lambda_{2} \lambda_{3}\right)^{2}-1\right) \\
& \left.+\lambda_{1} \lambda_{2} \lambda_{3}\right] \cdot \\
& \left.+\left(\frac{\left(\lambda_{1} \lambda_{2} \lambda_{3}\right)^{2}-1}{2}-\log \lambda_{1} \lambda_{2} \lambda_{3}\right)^{3}\right]+\frac{1}{\lambda_{2}}[1 \\
& \left.+\lambda_{3} \lambda_{2} \lambda_{3}\right] \\
& \left.+\lambda_{1} \lambda_{2} \lambda_{3} \log \left(1-\frac{1}{\lambda_{1} \lambda_{2} \lambda_{3}}\right)+\frac{\chi}{\lambda_{1} \lambda_{2} \lambda_{2} \lambda_{3}}\right)+\frac{\mu}{\lambda_{1} \lambda_{2} \lambda_{3}}-\frac{\mu}{K T} \\
& \hline T / v
\end{aligned}
$$

Consider the shape of cucumber is cylindrical, so the stretches are represented by

$$
\begin{aligned}
& \lambda_{1}=\frac{l}{L}=\lambda_{L}, \\
& \lambda_{2}=\lambda_{3}=\frac{r}{R}=\lambda_{R} .
\end{aligned}
$$



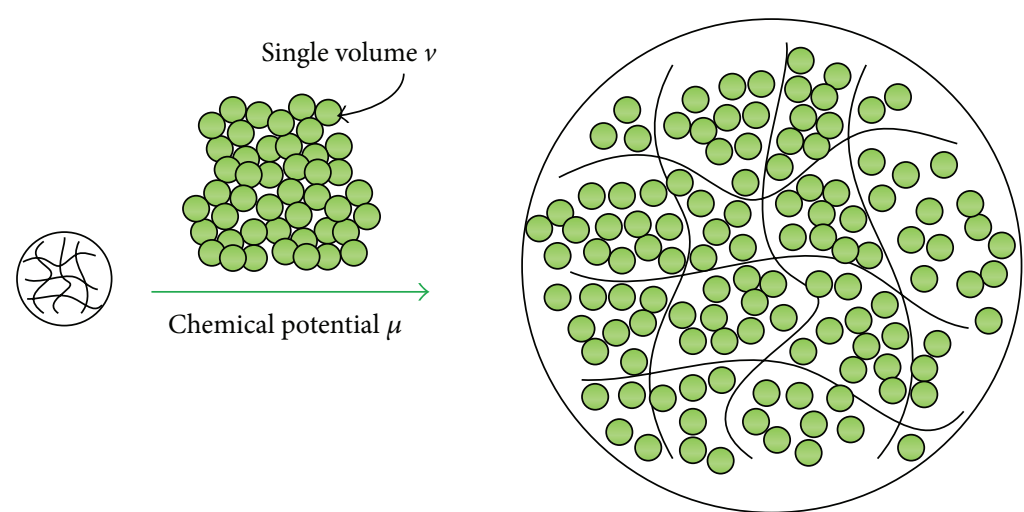

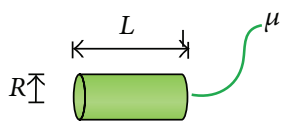

(a) Initial state

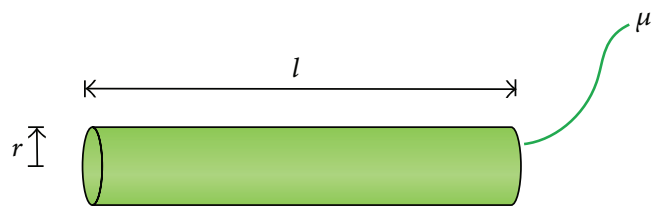

(b) Harvested state

FIgURE 2: The growth of a cucumber is analogous to the process of swelling in a tubular gel. Subjected to the chemical potential, the dry polymer network imbibes solvent molecules, forming a swollen gel. The sketched model characterizes the shape change of cucumber during growth, with the dimension enlarging from (a) $\pi R^{2} \times L$ at the initial state to (b) $\pi r^{2} \times l$ at the harvested state.

Thus, the constitutive equations are expressed as

$$
\begin{aligned}
& \frac{s_{L}}{K T / v}=N v\left[\frac{\lambda_{L}}{1-\left(\lambda_{L}^{2}+2 \lambda_{R}^{2}-3\right) / J_{m}}\right. \\
& \left.+\frac{2}{\lambda_{L}}\left(\lambda_{L}^{2} \lambda_{R}^{4}-1\right)\left(\frac{\lambda_{L}^{2} \lambda_{R}^{4}-1}{2}-\log \lambda_{L} \lambda_{R}^{2}\right)^{3}\right] \\
& +\frac{1}{\lambda_{L}}\left[1+\lambda_{L} \lambda_{R}^{2} \log \left(1-\frac{1}{\lambda_{L} \lambda_{R}^{2}}\right)+\lambda_{L} \lambda_{R}^{2}\right. \\
& \left.\quad-\frac{\mu}{K T} \lambda_{L} \lambda_{R}^{2}\right], \\
& \frac{s_{R}}{K T / v}=N v\left[\frac{\lambda_{R}}{1-\left(\lambda_{L}^{2}+2 \lambda_{R}^{2}-3\right) / J_{m}}\right. \\
& \left.+\frac{2}{\lambda_{2}}\left(\lambda_{L}^{2} \lambda_{R}^{4}-1\right)\left(\frac{\lambda_{L}^{2} \lambda_{R}^{4}-1}{2}-\log \lambda_{L} \lambda_{R}^{2}\right)^{3}\right] \\
& +\frac{1}{\lambda_{R}}\left[1+\lambda_{L} \lambda_{R}^{2} \log \left(1-\frac{1}{\lambda_{L} \lambda_{R}^{2}}\right)+\frac{\chi}{\lambda_{L} \lambda_{R}^{2}}\right. \\
& \left.-\frac{\mu}{K T} \lambda_{L} \lambda_{R}^{2}\right] .
\end{aligned}
$$

In cucumber growth, no external forces are applied to the biological entity, so that we model the growth of the cucumber as an isotropic free swelling process of the gel.
Therefore, we have $s_{L}=s_{R}=0$ and $\lambda_{L}=\lambda_{R}=\lambda$. Equations (5a) and (5b) are reduced to

$$
\begin{aligned}
& N v\left[\frac{\lambda}{1-3\left(\lambda^{2}-1\right) / J_{m}}\right. \\
& \left.+\frac{2}{\lambda}\left(\lambda^{6}-1\right)\left(\frac{\lambda^{3}-1}{2}-\log \lambda^{3}\right)^{3}\right]+\frac{1}{\lambda}[1 \\
& \left.+\lambda^{3} \log \left(1-\lambda^{-3}\right)+\frac{\chi}{\lambda^{3}}-\frac{\mu}{K T} \lambda^{3}\right]=0 .
\end{aligned}
$$

\section{Results and Discussion}

Figure 3 plots the swelling of four types of gel, differing in the material density of crosslink, $N v$, to characterize the growth of cucumbers. $J_{m}=100$ and $\chi=0.5$ as selected for representative values [6]. Gel, which is heavily cross-linked, is stiffer, behaved in mechanical strain responding. So $N v$ characterizes the stiffness of material, which, in terms of biological interpretation, is the species of cucumber featuring in strength, elasticity, and firmness. The growth stretch shows a nonlinear trend which is in good coincidence of observation of real cucumber growth; during the early stage, the cucumber enlarges slowly, which corresponds to the small stretch. When the chemical potential reaches a critical value, cucumber expands abruptly and then reaches a plateau for harvest. Some fruit is able to increase its volume by 100 times which resembles the performance of hydrogel in solvents absorbent. Figure 3 shows a continuous phase transition in material volume which recalls classic phase transformation in physics. For example, $\mathrm{PbZrO}_{3}$ ceramic transits from antiferroelectric state to ferroelectric state when the electric field exceeds the 


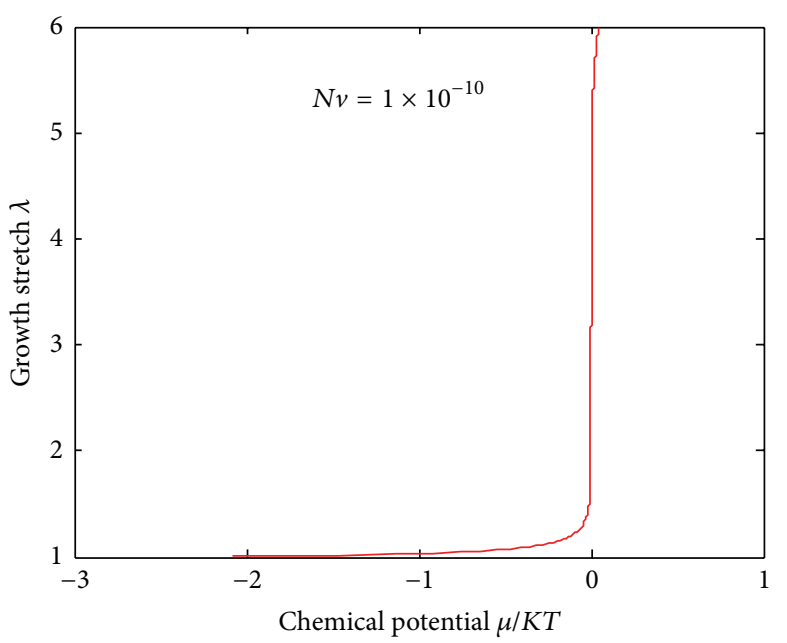

(a)

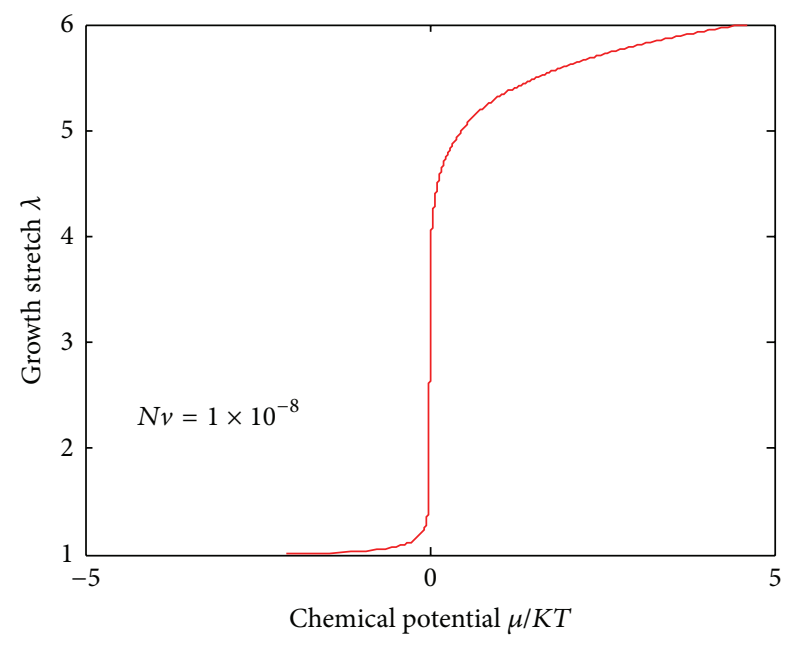

(c)

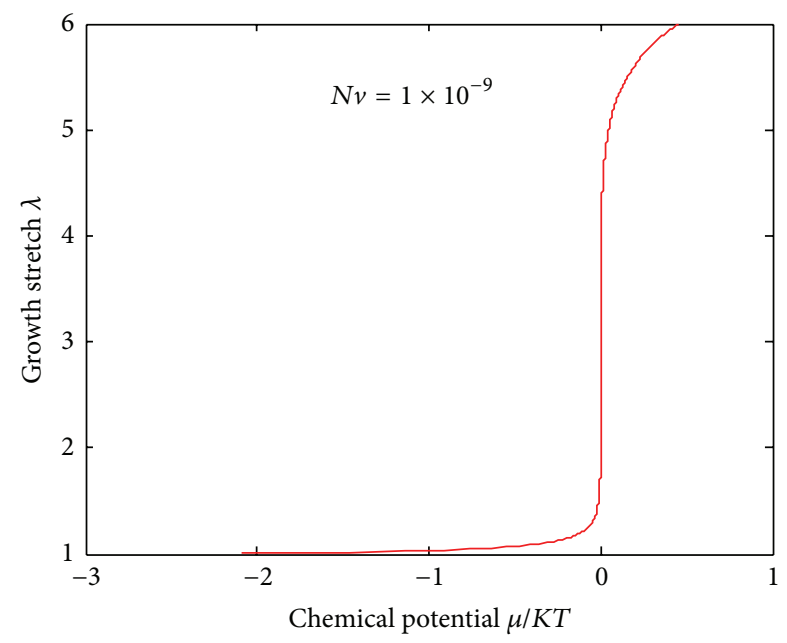

(b)

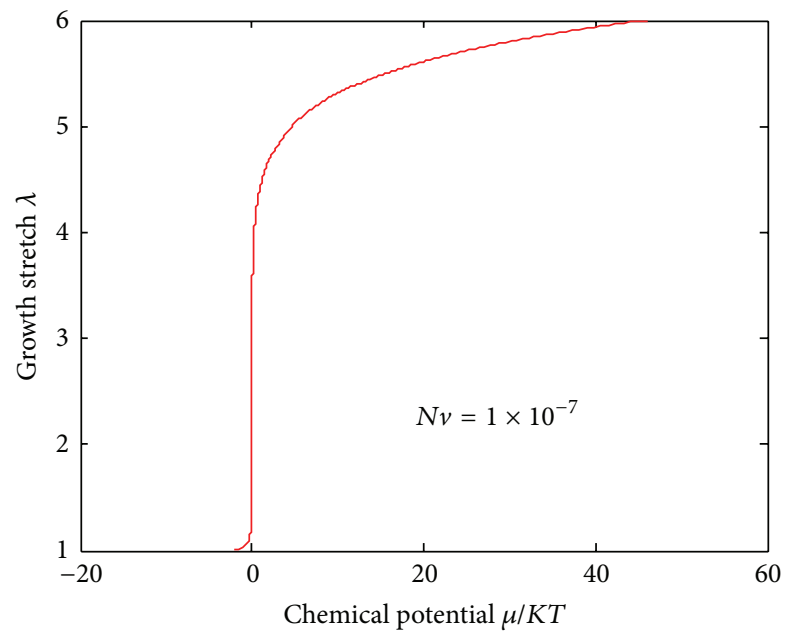

(d)

FIGURE 3: Stretch versus chemical potential during the gel swelling to model the growth of cucumber, showing an analogue to continuous phase transition.

critical magnitudes. In ferroelectric material, the transition is tunable by mechanical confinements such as stress or strain [12]. Here, we also investigate how the stiffness affects the cucumber growth. Figure 4 illustrates the swelling associated with crosslink density at selected chemical potentials. In Figures 4(c) and 4(d), the stretch curve is nonmonotonic that follows " $\mathrm{Z}$ " shape as crosslink density increases. At a specific $N v$ condition, two stretches coexist, which means that a special type of cucumber might grow with two shapes in the same body, at a prescribed hormone degree. Within a whole cucumber body, one part enables a fast and accelerated growth while the other grows normally. Figure 4(d) explains the news report in Figure 1 when the cucumber bulged locally in the tail. This verifies the first-order phase transition in thermodynamics. For example, at 0 degrees of temperature, $\mathrm{H}_{2} \mathrm{O}$ can coexist in both solid ice phase and liquid water phase.

We next investigate the phenomenon in Figure 1 in detail to extend the model to the study of postharvest process. After harvest, the cucumber is close to maturation but not fully ripened. To regulate the ripening speed, ethylene is spayed to form an artificial hormones environment. Denote the concentrate of ethylene as $\Omega$ and reexpress the free energy as

$$
\begin{aligned}
\frac{W}{N K T}= & -\frac{J_{m}}{2} \log \left(1-\frac{3 \lambda^{2}-3}{J_{m}}\right) \\
& +\frac{1}{2}\left(\frac{\lambda^{6}-1}{2}-\log \lambda^{3}\right)^{4} \\
& -\frac{1}{N v}\left[\left(\lambda^{3}-1\right) \log \frac{\lambda^{3}}{\lambda^{3}-1}+\frac{\chi}{\lambda^{3}}\right] \\
& -\frac{1}{N v} \frac{\Omega}{K T}\left(\lambda^{3}-1\right) .
\end{aligned}
$$

Figure 5 plots the free energy landscapes, normalized by NKT. Three magnitudes of crosslink density are prescribed with variation less than $10 \%$, but the associated energy curves 


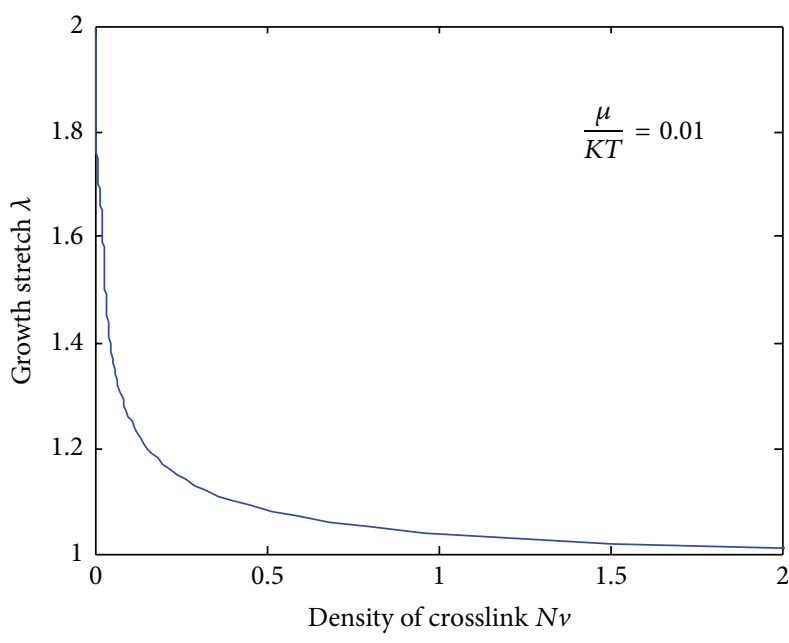

(a)

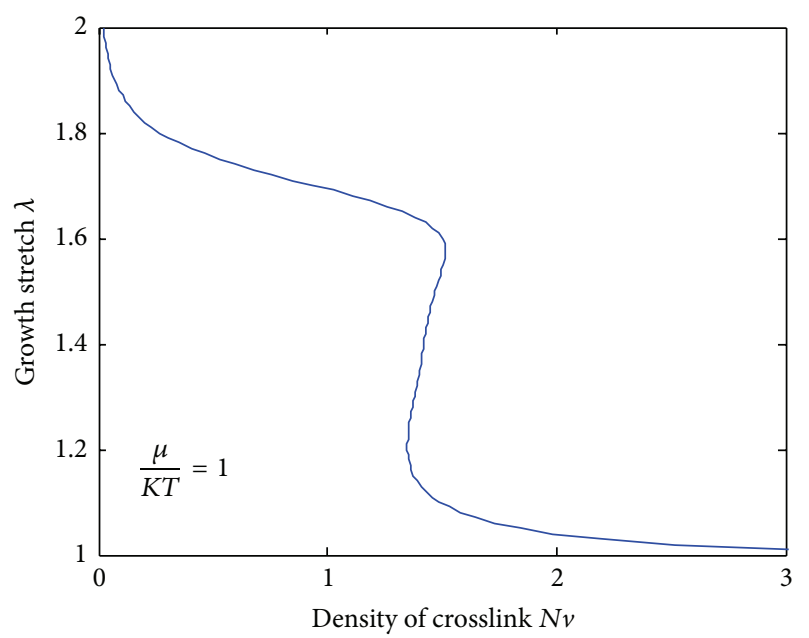

(c)

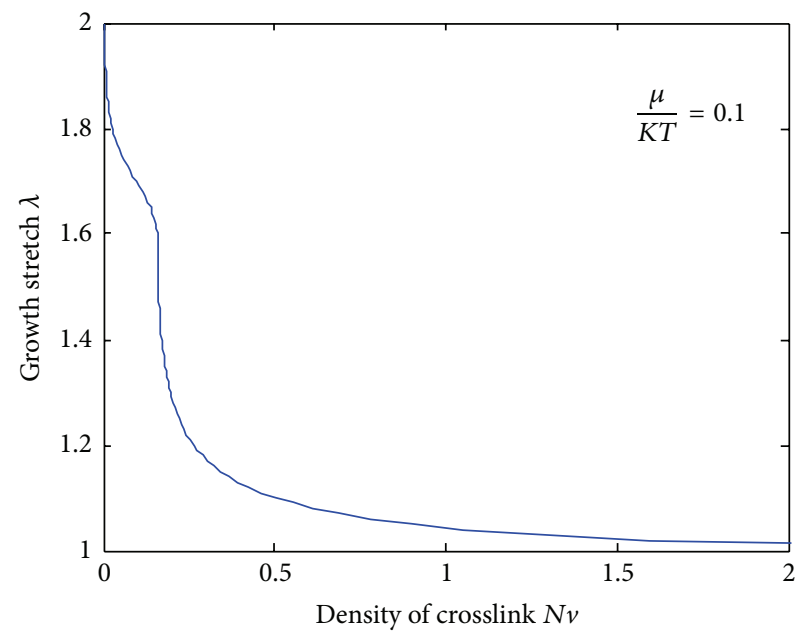

(b)

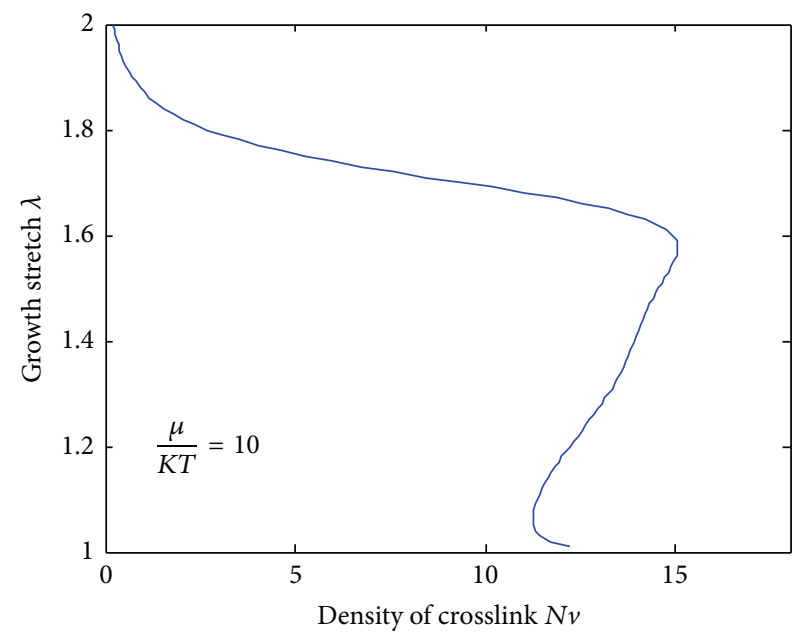

(d)

FIGURE 4: The growth processes by the model of gel swelling are influenced by chemical potential and density of crosslink, which are analogous to the concentration of plant hormone and cucumber stiffness in species. (a)-(d) show the effect of crosslink density, which denotes the firmness of cucumber species.

distinguish greatly in landscape. At $N v=11.2$, the thermodynamics system stabilizes at a lower stretch phase, since the energy minimizes. The higher stretch phase, corresponding to the second-minimized energy, is the metastable state that the gel might stabilize here temporally; but under a small perturbation, it could transit to the lower stretch state for global stabilization. Similar case is also presented in $N v=$ 11.4 since two minima are presented. The small stretch phase is metastable and large stretch phase is globally stable. Equation (7) offers to determine a level of density, $N v=$ 11.317, when the two stretches, $\lambda^{\prime}$ and $\lambda^{\prime \prime}$, share the same level of minimum free energy that both are globally stable. The two stretches in the growth of cucumber during postharvest process are possibly coexisting, only subject to a specific level of ethylene concentration.

Not all the cucumbers ripen with coexistence. The phenomenon is determined by the critical conditions of both the density of crosslink $N v$ and the chemical potential $\Omega / K T$. Here, Figure 6(a) shows the mechanism. At the onset of phase coexistence of $\lambda^{\prime}$ and $\lambda^{\prime \prime}$, the critical density $N v^{*}$ separates the curve, resulting in two grey regions which are the same in area. In thermodynamics, this line is defined as Maxwell line [13]. Following the classic theory in physics, we denote the critical value as Maxwell density to study the phase behavior in the current biochemical system.

The critical Maxwell density is a function of chemical potential that determines the occurrence for transition. By solving equations in Appendix, we plot in Figure 6(b) the relations. Under a prescribed chemical ethylene concentrate, cucumbers with density above the critical line are stable in the rod phase (phase B) with a small growth stretch; for cucumbers with density below the critical line, they bulge (phase A) for stabilization. For cucumbers holding the density that exactly falls in the critical line, their tails bulge 


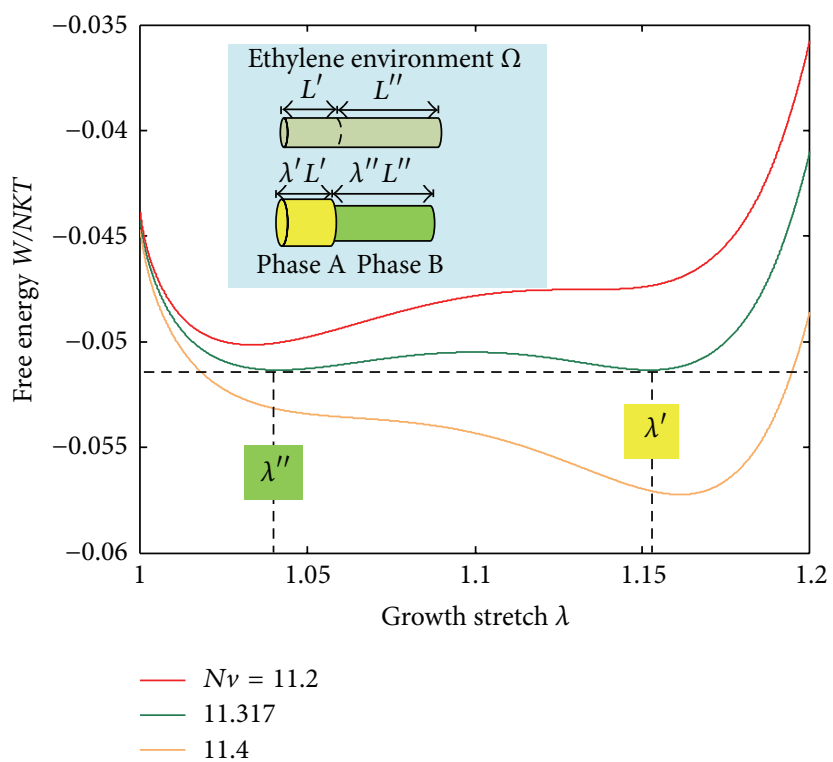

FIGURE 5: Coexistence of two stretch phases of $\lambda^{\prime}=1.15$ and $\lambda^{\prime \prime}=1.04$ subject to the conditions of $\Omega / K T=10$ and $N v=11.317$.

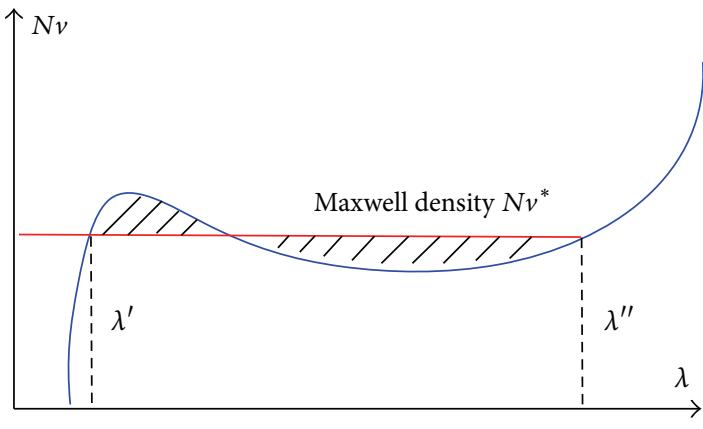

(a)

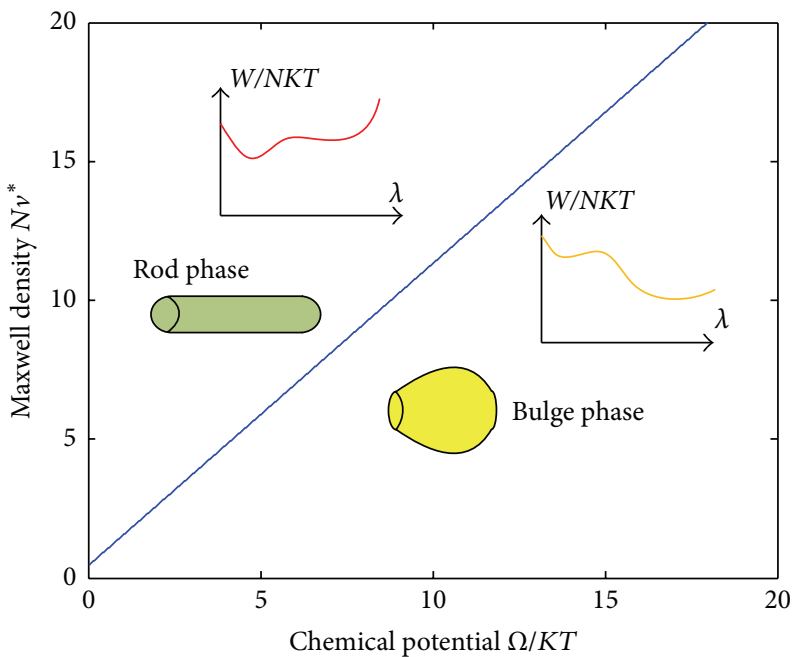

(b)

Figure 6: (a) The Maxwell density determines the coexistence of stretch states where the two states have the equal grey area. (b) Above the critical value, the small growth stretch state is stable; below the value, the large stretch state is stable.

but bodies are left in rod state. The local bulging instability has been documented in the research of tissue engineering, that is, aneurysm angiogenesis [14-16], with extensive solution of the inhomogeneous deformation. Here, we explain the mechanism for the first time from the perspective of botany in biology and characterize it using chemical thermodynamics. Using this model, an engineer is able to regulate the growth of plant and to achieve a prescribed shape in fruits and vegetables [17]. This offers a new insight into on-demand manufacturing. The element in mechanical structure is fabricated in a biological way to form its profile naturally.

\section{Conclusion}

In summary, the growth of cucumber is analogous to the swelling of gel. We characterize the phenomena of cucumber growth, covering the processes of both preharvest and postharvest, by establishing a thermodynamic gel model. The phase behavior in plant growth resembles the transition in ferroelectric ceramic and other biological tissue growth. The model offers solutions to regulate fruit development and can be extended to other plant candidates for potential technique in biological and food engineering. 


\section{Appendix}

Assume the cucumber gel is composed of two parts with lengths $L^{\prime}$ and $L^{\prime \prime}$, with $L^{\prime}+L^{\prime \prime}=L$. At the phase coexistence, the bulge phase is of length $\lambda^{\prime} L^{\prime}$ and the rod phase is of length $\lambda^{\prime \prime} L^{\prime \prime}$. The areas are $A^{\prime}$ and $A^{\prime \prime}$. Define $L^{\prime} / L=\alpha$ and $L^{\prime \prime} / L=1-\alpha$. The free energy is then expressed as $\Pi=A^{\prime} L^{\prime} W\left(\lambda^{\prime}\right)+A^{\prime \prime} L^{\prime \prime} W\left(\lambda^{\prime \prime}\right)$. The variation of energy is

$$
\begin{aligned}
\delta \Pi= & A^{\prime} W\left(\lambda^{\prime}\right) \delta L^{\prime}+A^{\prime} L^{\prime} \frac{\partial W\left(\lambda^{\prime}\right)}{\partial \lambda^{\prime}} \delta \lambda^{\prime} \\
& +A^{\prime \prime} W\left(\lambda^{\prime \prime}\right) \delta L^{\prime \prime}+A^{\prime \prime} L^{\prime \prime} \frac{\partial W\left(\lambda^{\prime \prime}\right)}{\partial \lambda^{\prime \prime}} \delta \lambda^{\prime}
\end{aligned}
$$

which gives

$$
\begin{aligned}
\frac{\delta \Pi}{A L}= & \lambda^{\prime 2} W\left(\lambda^{\prime}\right) \delta\left(\frac{L^{\prime}}{L}\right)+\lambda^{\prime 2} \frac{L^{\prime}}{L} \frac{\partial W\left(\lambda^{\prime}\right)}{\partial \lambda^{\prime}} \delta \lambda^{\prime} \\
& +\lambda^{\prime \prime 2} W\left(\lambda^{\prime \prime}\right) \delta\left(\frac{L^{\prime \prime}}{L}\right) \\
& +\lambda^{\prime \prime}\left(\frac{L^{\prime \prime}}{L}\right) \frac{\partial W\left(\lambda^{\prime \prime}\right)}{\partial \lambda^{\prime \prime}} \delta \lambda^{\prime},
\end{aligned}
$$

and rewritten as

$$
\begin{aligned}
\frac{\delta \Pi}{A L}= & \lambda^{\prime 2} W\left(\lambda^{\prime}\right) \delta \alpha+\alpha \lambda^{\prime 2} \frac{\partial W\left(\lambda^{\prime}\right)}{\partial \lambda^{\prime}} \delta \lambda^{\prime} \\
& -\lambda^{\prime \prime 2} W\left(\lambda^{\prime \prime}\right) \delta \alpha+(1-\alpha) \lambda^{\prime \prime 2} \frac{\partial W\left(\lambda^{\prime \prime}\right)}{\partial \lambda^{\prime \prime}} \delta \lambda^{\prime \prime} \\
= & \alpha \lambda^{\prime 2} \frac{\partial W\left(\lambda^{\prime}\right)}{\partial \lambda^{\prime}} \delta \lambda^{\prime}+(1-\alpha) \lambda^{\prime \prime 2} \frac{\partial W\left(\lambda^{\prime \prime}\right)}{\partial \lambda^{\prime \prime}} \delta \lambda^{\prime \prime} \\
& +\left[\lambda^{\prime 2} W\left(\lambda^{\prime}\right)-\lambda^{\prime \prime 2} W\left(\lambda^{\prime \prime}\right)\right] \delta \alpha .
\end{aligned}
$$

According to the second law of thermodynamics, the system is stable by minimizing the energy change for any arbitrary variation of quantities, so we have

$$
\begin{aligned}
\alpha \lambda^{\prime 2} \frac{\partial W\left(\lambda^{\prime}\right)}{\partial \lambda^{\prime}} & =0 \\
(1-\alpha) \lambda^{\prime \prime 2} \frac{\partial W\left(\lambda^{\prime \prime}\right)}{\partial \lambda^{\prime \prime}} & =0 \\
\lambda^{\prime 2} W\left(\lambda^{\prime}\right)-\lambda^{\prime \prime 2} W\left(\lambda^{\prime \prime}\right) & =0
\end{aligned}
$$

\section{Acknowledgments \\ This research was supported by the National Natural Science Foundation of China (no. 11402184), Shaanxi Province, and China Postdoctoral Science Foundation (no. 2014M560761).}

Substituting the specific Gent and Flory-Rehner energy functions in (A.4), we obtain

$$
\begin{aligned}
& N K T(1-\alpha)\left[\frac{\lambda^{\prime \prime 3}}{1-3\left(\lambda^{\prime \prime 2}-1\right) / J_{m}}\right. \\
& \left.+2 \lambda^{\prime \prime}\left(\lambda^{\prime \prime 6}-1\right)\left(\frac{\lambda^{\prime \prime 3}-1}{2}-\log \lambda^{\prime \prime 3}\right)^{3}\right] \\
& +\frac{K T}{v}(1-\alpha) \lambda^{\prime \prime}\left[1+\lambda^{\prime \prime 3} \log \left(1-\lambda^{\prime \prime-3}\right)+\frac{\chi}{\lambda^{\prime \prime 3}}\right. \\
& \left.-\frac{\mu}{K T} \lambda^{\prime \prime 3}\right]=0 \\
& N K T \alpha\left[\frac{\lambda^{\prime 3}}{1-3\left(\lambda^{\prime 2}-1\right) / J_{m}}\right. \\
& \left.+2 \lambda^{\prime}\left(\lambda^{\prime 6}-1\right)\left(\frac{\lambda^{\prime 3}-1}{2}-\log \lambda^{\prime 3}\right)^{3}\right]+\frac{K T}{v} \\
& \cdot \alpha \lambda^{\prime}\left[1+\lambda^{\prime 3} \log \left(1-\lambda^{\prime-3}\right)+\frac{\chi}{\lambda^{\prime 3}}-\frac{\mu}{K T} \lambda^{\prime 3}\right] \\
& =0 \text {, } \\
& -\lambda^{\prime 2} \frac{N K T}{2} J_{m} \log \left(1-3 \frac{\lambda^{\prime 2}-1}{J_{m}}\right)+\lambda^{\prime 2} \\
& \cdot \frac{N K T}{2}\left(\frac{\lambda^{\prime 6}-1}{2}-\log \lambda^{\prime 3}\right)^{4}-\lambda^{\prime 2} \\
& \frac{K T}{v}\left[\left(\lambda^{\prime 3}-1\right) \log \frac{\lambda^{\prime 3}}{\lambda^{\prime 3}-1}+\frac{\chi}{\lambda^{\prime 3}}\right]-\lambda^{\prime 2} \frac{\Omega}{v}\left(\lambda^{\prime 3}\right. \\
& -1)=-\lambda^{\prime \prime 2} \frac{N K T}{2} J_{m} \log \left(1-3 \frac{\lambda^{\prime \prime 2}-1}{J_{m}}\right)+\lambda^{\prime 2} \\
& \frac{N K T}{2}\left(\frac{\lambda^{\prime \prime 6}-1}{2}-\log \lambda^{\prime \prime 3}\right)^{4}-\lambda^{\prime \prime 2} \\
& \frac{K T}{v}\left[\left(\lambda^{\prime \prime 3}-1\right) \log \frac{\lambda^{\prime \prime 3}}{\lambda^{\prime \prime 3}-1}+\frac{\chi}{\lambda^{\prime \prime 3}}\right]-\lambda^{\prime \prime 2} \\
& \cdot \frac{\Omega}{v}\left(\lambda^{\prime \prime 3}-1\right) \text {. }
\end{aligned}
$$

Solving (A.5), we are able to determine the critical conditions for phase coexistence.

\section{Competing Interests}

The authors declare that they have no competing interests. 
Bo Li acknowledges XJTU for startup fund and International Collaboration Project Fund.

\section{References}

[1] B. M. Hurr, D. J. Huber, C. E. Vallejos, E. Lee, and S. A. Sargent, "Ethylene-induced overproduction of reactive oxygen species is responsible for the development of watersoaking in immature cucumber fruit," Journal of Plant Physiology, vol. 170, no. 1, pp. 56-62, 2013.

[2] S. J. Gerbode, J. R. Puzey, A. G. McCormick, and L. Mahadevan, "How the cucumber tendril coils and overwinds," Science, vol. 337, no. 6098, pp. 1087-1091, 2012.

[3] Y. Forterre, "Slow, fast and furious: understanding the physics of plant movements," Journal of Experimental Botany, vol. 64, no. 15, pp. 4745-4760, 2013.

[4] W. Hong, Z. Liu, and Z. Suo, "Inhomogeneous swelling of a gel in equilibrium with a solvent and mechanical load," International Journal of Solids and Structures, vol. 46, no. 17, pp. 32823289, 2009.

[5] L. Jin, S. Cai, and Z. Suo, "Creases in soft tissues generated by growth," EPL, vol. 95, no. 6, Article ID 64002, 2011.

[6] Z. Liu, S. Swaddiwudhipong, and W. Hong, "Pattern formation in plants via instability theory of hydrogels," Soft Matter, vol. 9, no. 2, pp. 577-587, 2013.

[7] J. Yin, G. J. Gerling, and X. Chen, "Mechanical modeling of a wrinkled fingertip immersed in water," Acta Biomaterialia, vol. 6, no. 4, pp. 1487-1496, 2010.

[8] B. Li, F. Jia, Y.-P. Cao, X.-Q. Feng, and H. Gao, "Surface wrinkling patterns on a core-shell soft sphere," Physical Review Letters, vol. 106, no. 23, Article ID 234301, 2011.

[9] S. Cai and Z. Suo, "Mechanics and chemical thermodynamics of phase transition in temperature-sensitive hydrogels," Journal of the Mechanics and Physics of Solids, vol. 59, no. 11, pp. 22592278, 2011.

[10] M. C. Boyce and E. M. Arruda, "Constitutive models of rubber elasticity: a review," Rubber Chemistry and Technology, vol. 73, no. 3, pp. 504-523, 2000.

[11] P. J. Flory and J. Rehner Jr., "Statistical mechanics of cross-linked polymer networks II. Swelling," The Journal of Chemical Physics, vol. 11, no. 11, pp. 521-526, 1943.

[12] S. E. Young, J. Y. Zhang, W. Hong, and X. Tan, "Mechanical selfconfinement to enhance energy storage density of antiferroelectric capacitors," Journal of Applied Physics, vol. 113, no. 5, Article ID 054101, 2013.

[13] Y. A. Cengel, M. A. Boles, and M. Kanoğlu, Thermodynamics: An Engineering Approach, McGraw-Hill, New York, NY, USA, 2011.

[14] A. N. Gent, "Elastic instabilities in rubber," International Journal of Non-Linear Mechanics, vol. 40, no. 2-3, pp. 165-175, 2005.

[15] A. A. Alhayani, J. Rodríguez, and J. Merodio, "Competition between radial expansion and axial propagation in bulging of inflated cylinders with application to aneurysms propagation in arterial wall tissue," International Journal of Engineering Science, vol. 85, pp. 74-89, 2014.

[16] C. J. Cyron, J. S. Wilson, and J. D. Humphrey, "Mechanobiological stability: a new paradigm to understand the enlargement of aneurysms?" Journal of the Royal Society Interface, vol. 11, Article ID 20140680, 2014.

[17] X. Chen and J. Yin, "Buckling patterns of thin films on curved compliant substrates with applications to morphogenesis and three-dimensional micro-fabrication," Soft Matter, vol. 6, no. 22, pp. 5667-5680, 2010. 

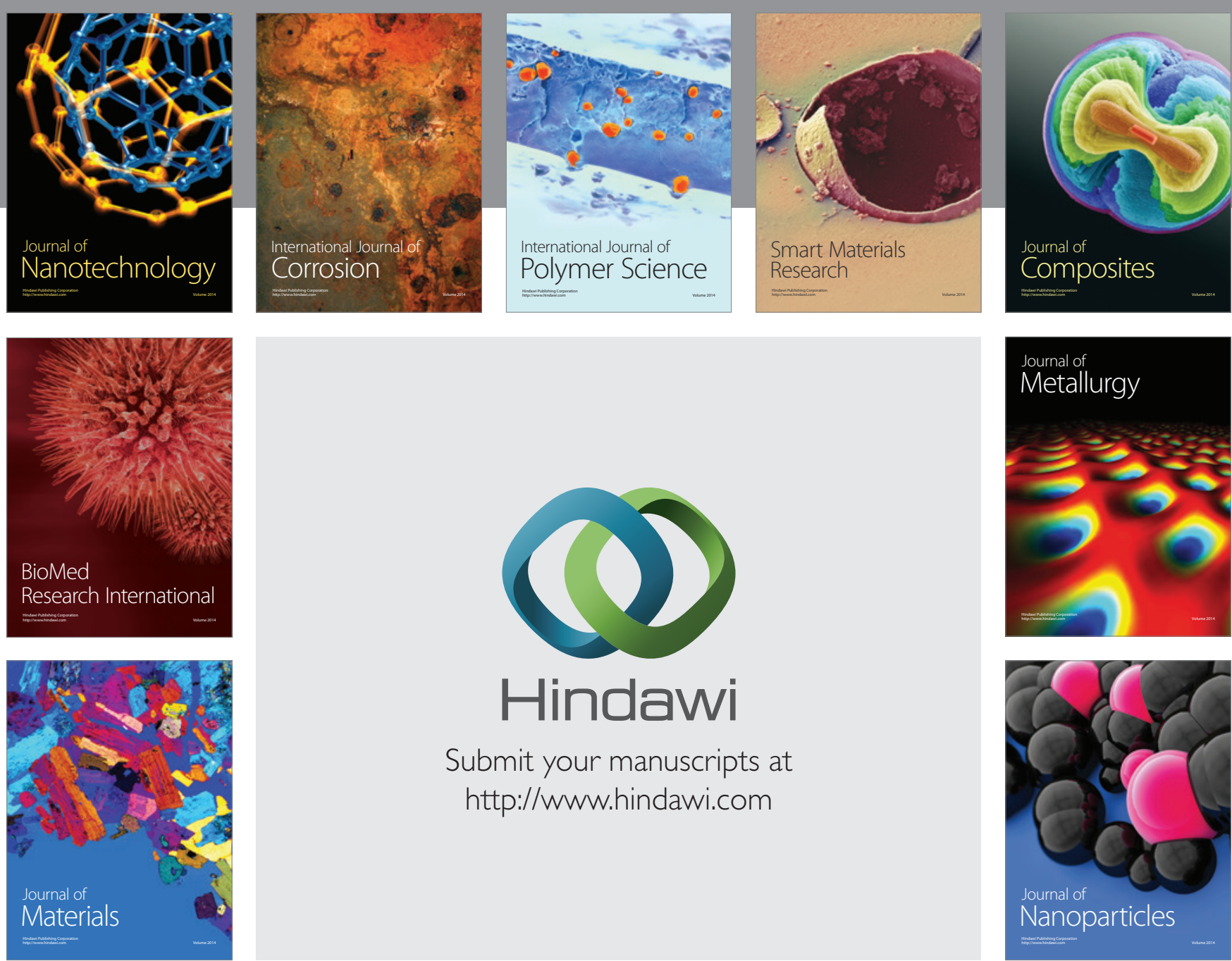

\section{Hindawi}

Submit your manuscripts at

http://www.hindawi.com

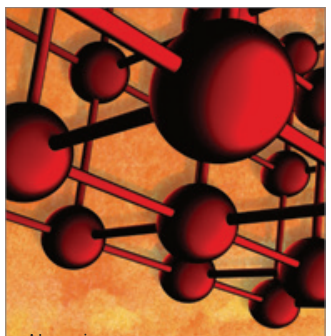

Materials Science and Engineering
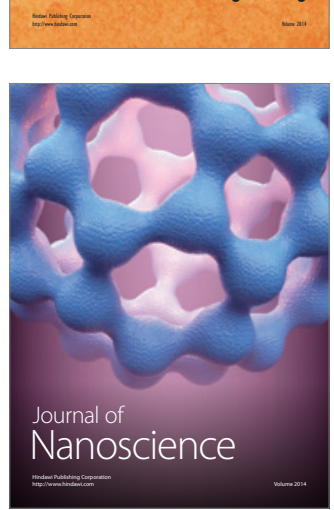
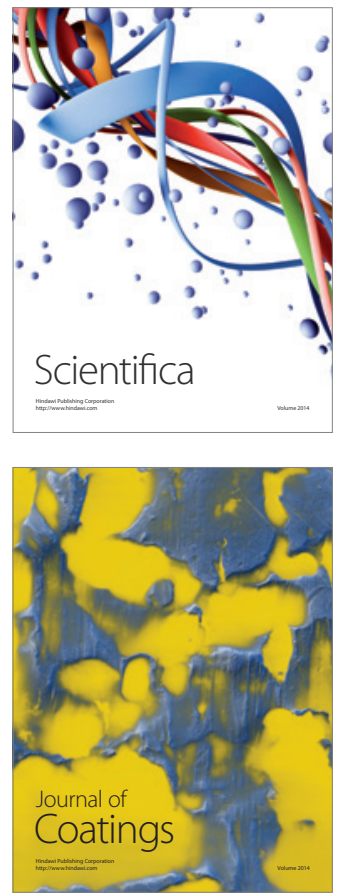
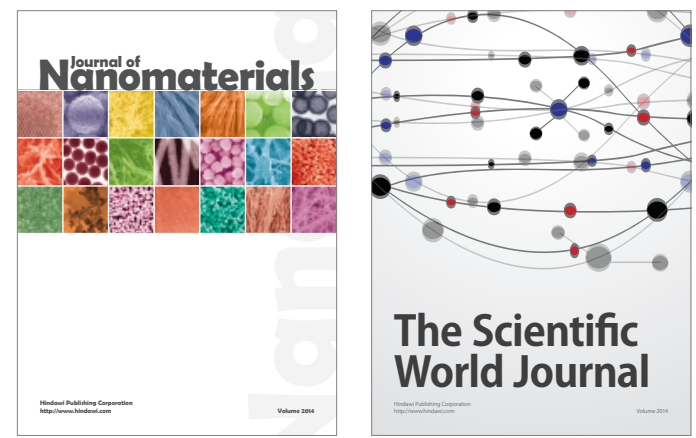

The Scientific World Journal
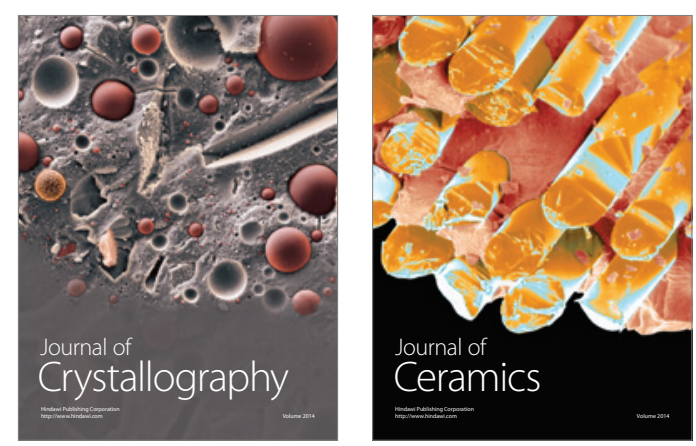
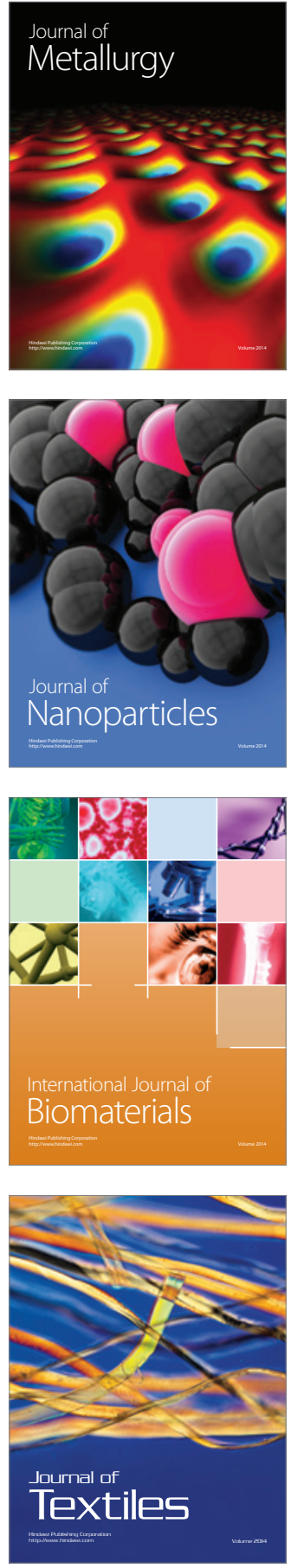